Article

\title{
Development of a Piezoelectric-Based Odor Reproduction System
}

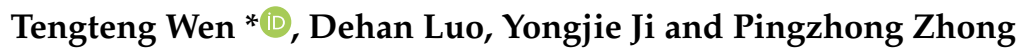 \\ School of Information Engineering, Guangdong University of Technology, Guangzhou 510006, China \\ * Correspondence: wentt.cn@outlook.com
}

Received: 15 July 2019; Accepted: 1 August 2019; Published: 6 August 2019

check for updates

\begin{abstract}
Odor reproduction, a branch of machine olfaction, is a technology through which a machine represents various odors by blending several odor sources in different proportions and releases them. In this paper, an odor reproduction system is proposed. The system includes an atomization-based odor dispenser using 16 micro-porous piezoelectric transducers. The authors propose the use of an electronic nose combined with a Principal Component Analysis-Linear Discriminant Analysis (PCA-LDA) model to evaluate the effectiveness of the system. The results indicate that the model can be used to evaluate the system.
\end{abstract}

Keywords: machine olfaction; odor reproduction; micro-porous piezoelectric transducers

\section{Introduction}

The term machine olfaction is usually referred to as a technology with which an intelligent machine detects and identifies gases or odors. An electronic nose is a gas/odor identification machine which is comprised of a gas sensor array and an intelligent identification block [1]. However, machine olfaction should embrace a larger area, in which the subject includes not only gas/odor identifications but also odor representations. In computer vision, colors (images) are captured by cameras and reproduced by displayers or printers. In computer audition, voices are sampled by recorders and played by speakers. It is analogous that machine olfaction includes both intelligent sensation and representation of odors. In other words, an odor identification instrument (electronic nose) 'smells' and records odors, and an odor representation (odor reproduction or olfactory display) machine reproduces odors. The term odor reproduction and the term olfactory display are different, because they generate odors differently. An odor reproduction instrument blends several odor sources then releases them, in which the blending process utilizes basic components either in a liquid or gas state. An olfactory display releases an odor from one of those well-prepared odor sources. It is reasonable to assume that the number of odors reproduced by an odor reproduction instrument is bigger than an olfactory display if the number of channels are the same.

Many studies concerning olfactory display have been reported in recent decades. Morton L. Heilig et al. invented an olfactory display called the "sensorama simulator", in which odor-stimulating substances were released from a specially designed chair in the 1960s [2]. This was probably the first report of an olfactory display. Y. Yanagida et al. developed a nose-tracing air cannon which was capable of tracing human noses and emitting odors [3]. D. W. Kim et al. designed an aroma-card based olfactory display [4]. D. Noguchi et al. developed a multi-channel olfactory display which was based on ink-jet technology [5]. S. H. Abid et al. proposed a multimedia decoder system with an olfactory display that contained six micro-porous piezoelectric transducers [6]. T. Nakamoto et al. developed an olfactory display based on surface acoustic wave (SAW) technology [7]. J. A. Covington et al. developed a multi-channel portable olfactory display system which consisted of eight thermalization-based odor generators controlled by a pulse-width modulation (PWM) approach [8]. 
Atomization and thermalization are two solutions for building an odor reproduction instrument or an olfactory display. Atomization is an approach to dispense odorants with the advantages that the dispensing process is fast and the dispensing quantity is controllable. Surface acoustic wave (SAW) transducers are a way of atomizing odorants. Liquid on a piezoelectric substrate is vibrated and atomized by a spurred wave generated by an interdigital transducer electrode [9]. A number of studies on SAW-based olfactory displays have been reported $[7,10,11]$. The other form of atomization-based olfactory display is based on micro-porous piezoelectric transducers [6]. A micro-porous piezoelectric transducer atomizes liquid by means of a metal mesh smashing the liquid. The smash of the metal mesh is driven by the vibration of a piezoelectric substrate. Piezoelectric transducers are suitable for commercial uses, due to their advantage of low cost. Thermalization is the other way of dispensing odors, by vaporizing odor sources in the liquid state or the solid state using PWM heaters $[8,12]$. A temperature controller for odor dispensers should be embedded in a thermalization-based odor reproduction instrument or olfactory display to avoid the possibility of scorching odor sources.

The advance of odor reproduction is slow, and few studies have been reported. A study on the development of an odor reproduction system was reported by T. Nakamoto et al. [13-15]. The system consisted of an odor identification instrument (sensor array or portable mass spectrometer) and an odor blender. The blender reproduced odors according to the measurement data recorded by the odor identification instrument. The obstacles to building an odor reproduction system are as follows: Firstly, odor blending should be controlled precisely, because a mixture of various odorants in different proportions may change the odor. Secondly, the scheme of how to reproduce odors according to odor information is ambiguous. In the odor reproduction system proposed by T. Nakamoto et al., as illustrated above, the system was a kind of feedback loop which made reproduction a time-consuming process. D. Harel et al. proposed an odor communication system, in which an odor "sniffer" recorded the odor information and an odor "whiffer" reproduced the odor [16]. However, the coding of odor information is unpracticable for this kind of system, at present. Perfumery is assumed to be the most similar technique to odor reproduction, although various aspects differ from odor reproduction, such as aroma duration, stability, and so on. The scent of perfume can be divided into three stages: Top note, body note, and basic note, since a bottle of perfume is a combination of fragrance sources which possess different fragrance retention times. However, an odor reproduction system reproduces different odors dynamically. This means that those fragrance sources which have shorter retention times may be selected. Besides, perfumery is an art, to some extent, so flavorists may incorporate their background knowledge into perfume formulas; however, an odor reproduction system needs a more regularized set of rules for blending odor sources. Thirdly, the biological, neurological, and psychological mechanisms of odor perception are unclear as of yet. Stereochemistry and vibrational theory are considered to be the two acceptable theories that described relationships between odorants and odor qualities, although both theories have some exceptions that fail to describe such relationships fully [17]. Recent research has revealed that strong correlation between odor chemical features and perceptual qualities [18,19].

The potential applications of odor reproduction technology are manifold. Odor reproduction can be applied to various applications, such as virtual and augmented reality, smart homes, electronic commerce, and so on. Moreover, odor reproduction can be applied to some medical uses, such as the first-diagnosis of Parkinson's disease, as the degradation of olfaction is a representative characteristic of the disease [20]. It is reasonable that an odor reproduction system should have the following features:

1. Reproduces odors in real-time;

2. A limited number of odor sources are used;

3. Capacity for reproducing a large number of odors; and

4. Has a user-friendly interface.

Considering that there is, at present, no appropriate ready-made instrument for the research of odor reproduction, we have developed an odor reproduction system for our future studies of odor 
reproduction. We have also proposed a method for verifying the performance of the system, using an electronic nose. In the following sections, the development of the odor reproduction system will be illustrated first. After that, the authors propose a method for the evaluation of the system using a commercial electronic nose. Finally, a discussion of the system and odor reproduction is given.

\section{Odor Reproduction System}

\subsection{Architecture}

The proposed odor reproduction system (ORS) consists of a controller unit, an odor blender, and a user-friendly interactive application, as shown in Figure 1. The odor blender is comprised of an odor dispenser, two fans, and a chamber. The odor blender implements the process of reproducing odors by blending several odor sources, also known as fragrance sources. The odor dispenser consists of an array of 16 odor atomizers, based on micro-porous piezoelectric transducers, to atomize the odor source liquid. Two fans are used for the process of blending, releasing odors, and cleaning residual gases in the chamber. The controller unit consists of a ready-to-use development platform designed by Digilent Inc., two peripheral devices (a gas sensor component and a Bluetooth component), and a self-designed circuit for driving the piezoelectric transducers and fans. We used the fifth hyper-text markup language (HTML 5) to develop a user-friendly interactive application.

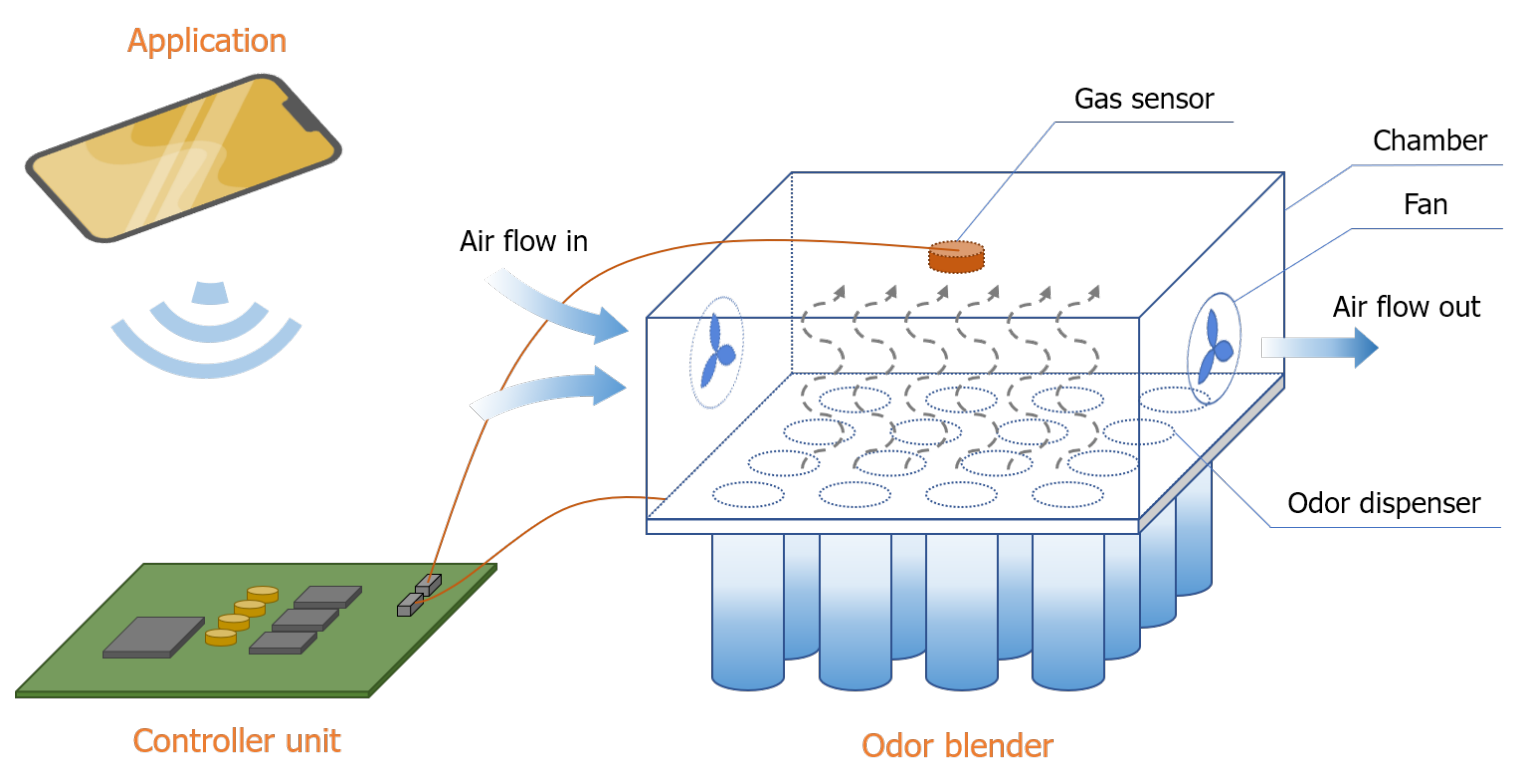

Figure 1. Structural schematic of the odor reproduction system.

\subsection{Odor Dispenser}

The odor dispenser is capable of releasing odorous compounds in certain proportions. It contains a $4 \times 4$ array of odor atomizers, where each odor atomizer consists of a micro-porous piezoelectric transducer, a plastic tube, and a cotton core, as shown in Figure 2a. The plastic tube stores an odor source and is screwed on the acrylic board, and the cotton core is applied for the absorption of the odor source liquid. The micro-porous piezoelectric transducer, as shown in Figure $2 b$, consists of a rubber gasket, a metal substrate, and a ring-shaped piezoelectric plate. The rubber gasket is used for isolation of the electric conduction from other conducting materials, and as a cushion against vibration. The metal substrate has a micro-porous metal mesh in the center, with a diameter of $35 \mathrm{~mm}$. The number of micro-pores in the metal mesh is approximately 700. Each micro-pore is a trumpet-shaped cylinder in which the upper cylindrical surface is smaller than the bottom, as shown in Figure $2 \mathrm{~b}$. A contact is attached to the piezoelectric plate, so that the power wire and ground wire can be connected between the piezoelectric plate and the metal substrate. A micro-porous piezoelectric 
transducer is driven by a wave with a frequency of around $113 \mathrm{kHz}$ and converts electric energy into kinetic energy due to inverse-piezoelectricity. The metal substrate vibrates along with the vibration of the ring-shaped piezoelectric plate, and the mesh in the center of the metal substrate smashes the liquid beneath the transducer. Some liquid flows through those micro-pores and is emitted in micro-droplet form.

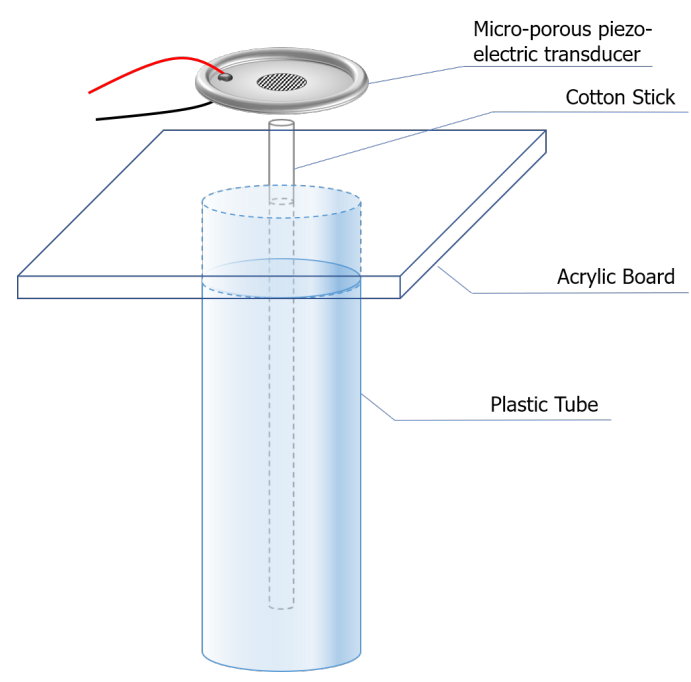

(a)

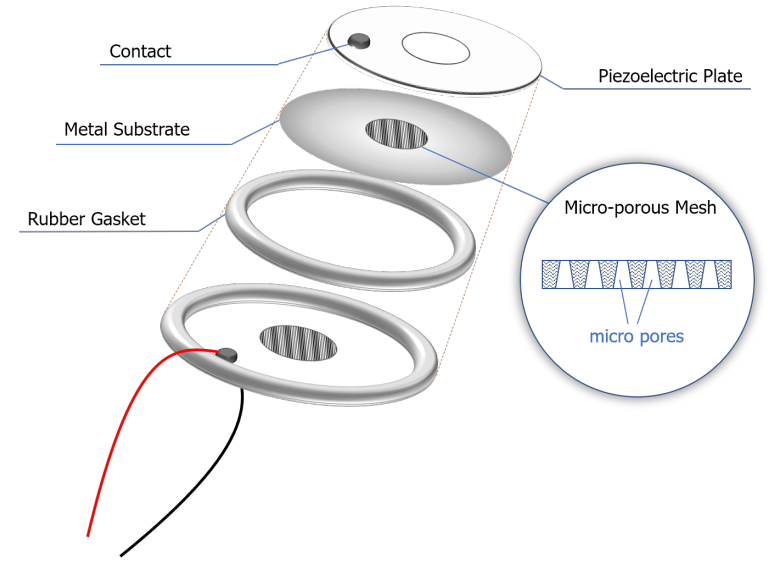

(b)

Figure 2. Odor dispenser. (a) An atomizer with a micro-porous piezoelectric transducer; and (b) a micro-porous piezoelectric transducer.

\subsection{Controller Unit}

The controller unit consists of a Digilent Arty Z7 development board with an all-programmable system on chip (SoC) manufactured by Xilinx, a self-designed board, a sensor component, and a Bluetooth component, as shown in Figure 3a. Multiple channels (16 channels) of driving wave signals, generated by the Arty Z7 development board, are amplified first by the self-designed circuit and, then, transmitted to the 16 micro-porous piezoelectric transducers.

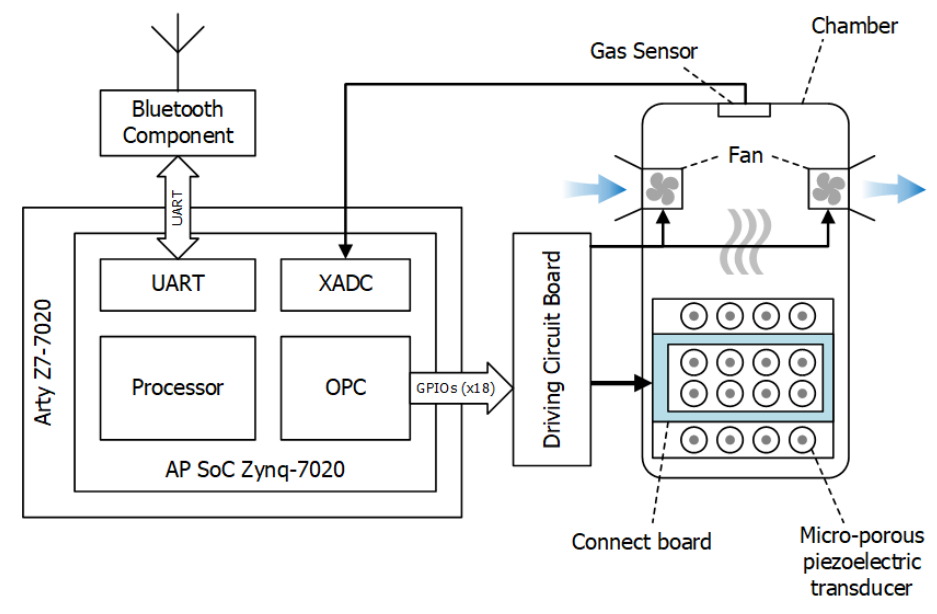

(a)

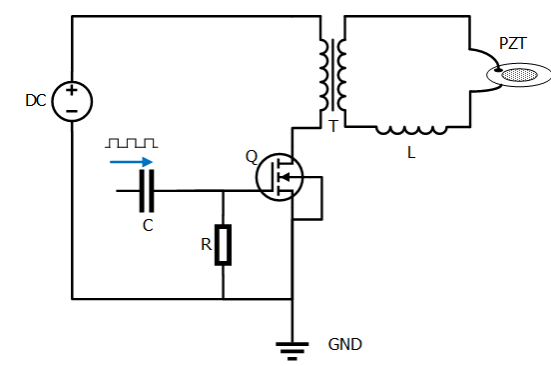

(b)

Figure 3. Hardware design. (a) Architecture of the odor reproduction system (ORS) design; and (b) the wave amplifying circuit. 


\subsubsection{Arty Z7-7020 Development Board}

The Digilent Arty Z7-7020 development board is a ready-to-use development platform designed around the Zynq-7000 ${ }^{\mathrm{TM}}$ All Programmable System-on-Chip (AP SoC) from Xilinx Inc. The Zynq platform is an easy-to-design platform with adequate resources to design a precise controlling module for the 16 piezoelectric atomizers. Some features of the development board are illustrated in Table 1.

Table 1. Some specifications of Arty Z7-7020 development board.

\begin{tabular}{ll}
\hline Item & Specification \\
\hline Product variant & Arty Z7-7020 \\
Zynq part & XC7Z020-1CLG400C \\
Look-up Tables (LUTs) & 53,200 \\
Flip-Flops & 106,400 \\
Block RAM & $630 \mathrm{~KB}$ \\
Clock Management Tiles & 4 \\
Processor & $650 \mathrm{MHz}$ dual-core Cortex-A9 processor \\
& $512 \mathrm{MB}$ DDR3 with 16-bit bus @ 1050 Mbps \\
Memory & $16 \mathrm{MB}$ Quad-SPI Flash with factory programmed 48-bit globally unique \\
& microSD slot \\
expansion connectors & Two standard Pmod ports \\
& Arduino/chipKIT Shield connector \\
\hline
\end{tabular}

\subsubsection{Driver Circuit}

The driver circuit is comprised of 16 amplifiers for the 16 atomizers, a fan driving circuit for the two fans, and a connect board. Each amplifier contains a transformer with 38 times amplification. The schematic of the amplifier circuit is shown in Figure 3b.

\subsubsection{Peripheral Devices}

There are two peripheral devices embedded in the system: A gas sensor component and a Bluetooth component. The gas sensor component, which contains a metal oxide sensor, TGS2600, Figaro Inc., was assembled on the roof of the chamber to measure the odor concentration. The gas component is intended for investigation of the atomization process, and so the gas sensor may be replaced by any other appropriate model. It is also used for the detection of whether the chamber is clean or not. The Bluetooth component is used for communication between the controller unit and the user-friendly interactive application software.

\subsection{Firmware}

The firmware integrates a processing system (PS) design and an odor blending controller (OBC) module in programmable logic (PL), as shown in Figure 4. The PS design includes several intellectual properties (IPs): An ARM dual-core Cortex A9 processor hardcore IP, a Xilinx analog mixed-signal IP (XADC), a general-purpose I/O (GPIO) IP, and an advanced extensible interface (AXI) block-RAM (BRAM) controller IP. All these modules are linked by an interconnect IP using AXI point-to-point channels. The analog input signal from the gas sensor component is converted into a digital signal by the XADC IP. The external Bluetooth component is connected to the processor hardcore IP directly by UART. The GPIO module is applied to register processing status in both PL and PS. The AXI BRAM controller is used for the processor to access the "Buffer" sub-module in the OBC module.

In PL, the OBC module receives messages from the processor, generates driving wave signals for some piezoelectric transducers, and controls the two fans using PWM signals. It has two operating modes: Chamber cleaning and blending. The "Buffer" module, which includes a BRAM IP, receives control messages from the processor. The "Parser" module parses and converts those control messages into control parameters for the "FSM", "Wave Controller", and "Fan Controller" modules. The "Wave Controller" module generates wave signals separately for those enabled channels. The duty ratios and 
frequencies can be modulated, and the periods of each channel can be configured. The "Fan Controller" module generates two PWM waves for the two fans. The fan controller module generates two PWM signals for the two external fans. A finite state machine (FSM) module implements the procedure of controlling the 16 micro-porous piezoelectric transducers and the two fans.

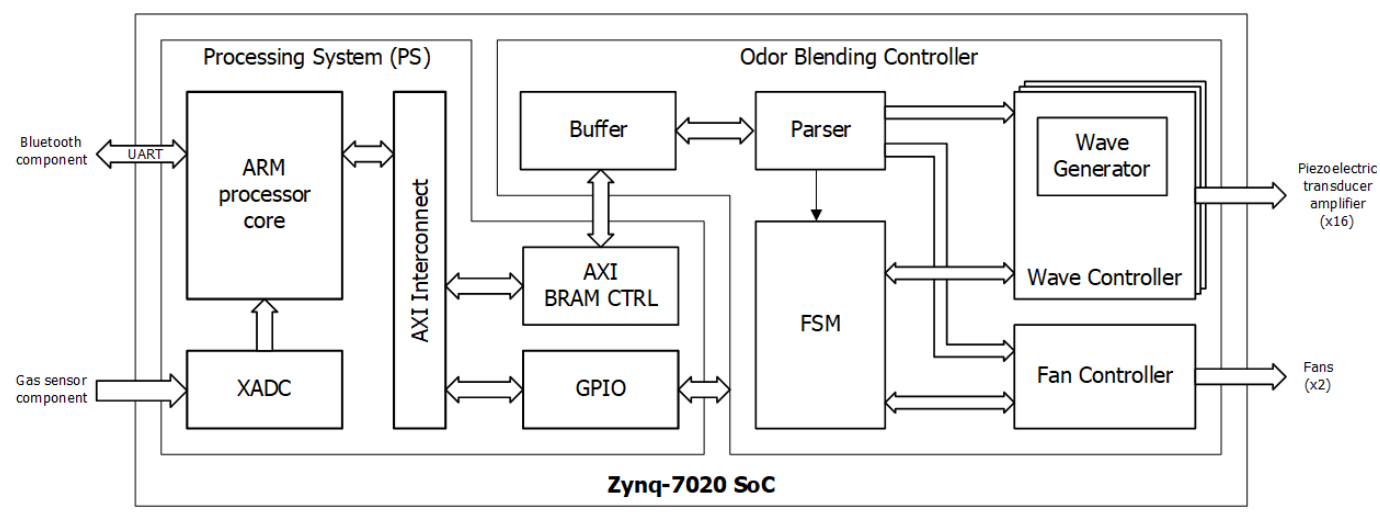

Figure 4. Firmware design in Zynq-7020.

The FSM implements precise control of odor reproduction, as shown in Figure 5. When the "Buffer" module receives a message from the processor, a register for indicating the reception of the message will be set to high, and the FSM jumps to the "PREPARE" state. The FSM enables the "Parser" module to read the whole message. After that, if a chamber cleanliness register is set high, the FSM will jump to the 'CLEAN' state. Otherwise, the FSM jumps to the "BLEND" state to start the blending process. In the "CLEAN" state, the "Fan Controller" module implements the cleaning of the chamber. In the "BLEND" state, the "Wave Controller" module enables and outputs wave signals for those channels used. Then, the FSM jumps to the 'RELEASE' state, where the "Fan Controller" module enables both fans by two PWM control signals. Finally, the FSM returns to 'IDLE' state.

\subsection{Software}

The software running in the Zynq platform implements several operations. First, it implements the communication of the device and the program running on the client-side. Second, it sends odor reproduction messages by writing the BRAM block in the OBC module. Third, the software converts the analog signal from the gas sensor to a digital signal. It also registers the cleanliness status of the chamber, if the response of the gas sensor is higher than a threshold value. Lastly, it collects status data and sends them to the user side for monitoring.

The other software is a user-friendly interactive application developed in the Wechat platform, called "mini-program", which is an HTML5-based program. Users can operate the program by scanning a QR code without any installation. The application has an interface with which one can configure the 'recipe' to produce an odor. It will be used for the study of odor reproduction methods. Figure 6 shows some of the interfaces of the mini-program. The mini-program has two functions: First, one can drag 16 seek bars to adjust the atomizing period separately for each channel. Second, when the mini-program is playing some videos, it will send messages to the odor blender to release some special odors; this functionality is currently under development. 


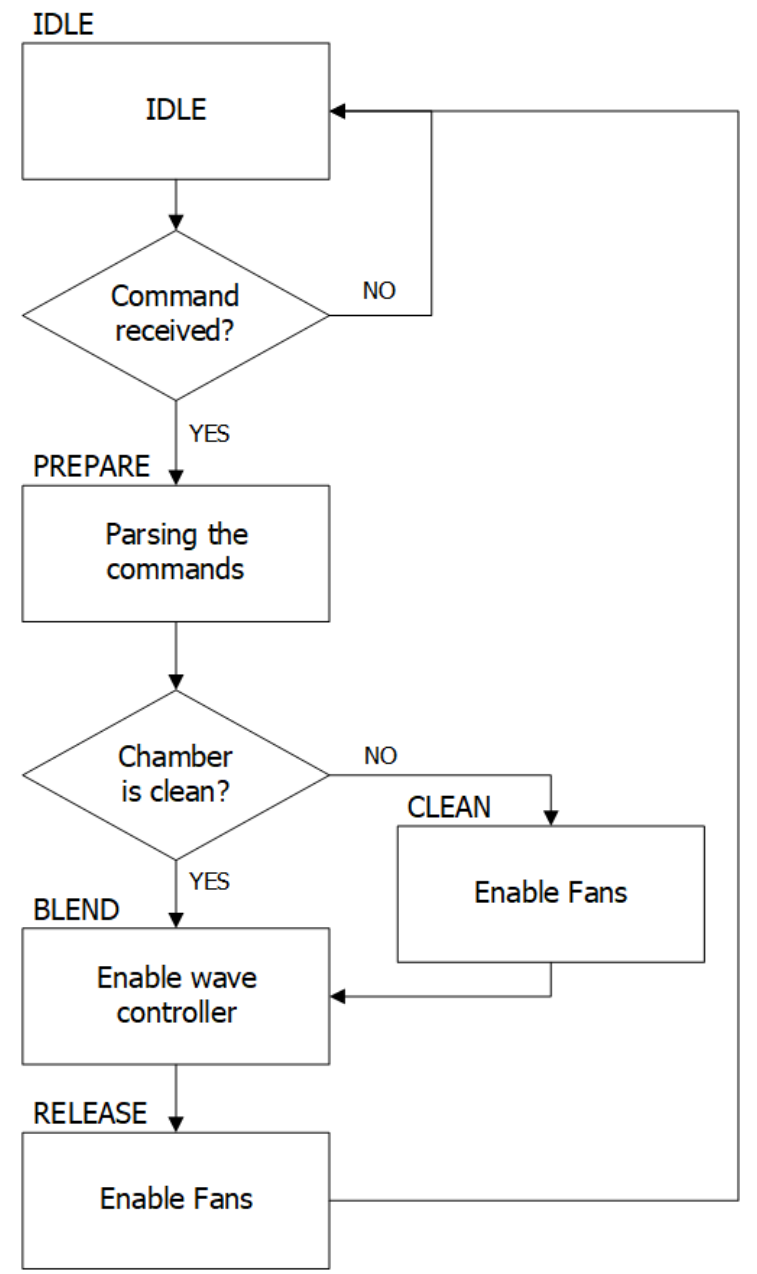

Figure 5. Finite state-machine of the firmware.
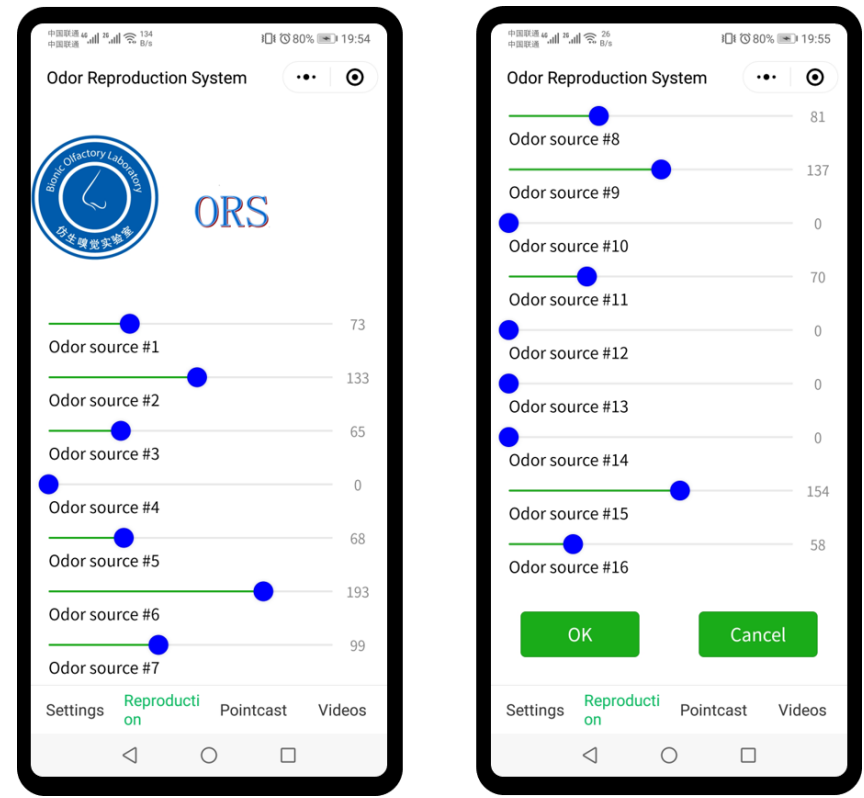

Figure 6. Mini-program for the odor reproduction system. 


\section{Evaluation of the System}

This experiment aims to verify the effectiveness of the proposed system reproducing an odor. The evaluation uses a linear classifier (PCA-LDA) with data sampled by a commercial electronic nose, PEN3, manufactured by Airsense Analytics GmbH. The training data were sampled from odors atomized by well-prepared mixed odor sources. The testing data were sampled from odors atomized by proportional blending (blending pure odor sources in various proportions). Four highly pure odor sources were used for the experiment; the sources used are shown in Table 2. Five circumstances of odor mixture with different proportions were randomly set, as shown in Table 3.

Table 2. Fragrance sources used in the experiment.

\begin{tabular}{lll}
\hline Index & Chemical & Manufacturer \\
\hline C1 & Ethanol & Shanghai Macklin Biochemical Co., Ltd. \\
C2 & Isopentylacetate & Chengdu Kelong Chemical Reagent Factory \\
C3 & Benzyl alcohol & Anhui Jinrong Flavor and Fragrance Co., Ltd. \\
C4 & Allyl cyclohexanepropionate & Penda Flavors and Fragrances (U.S.A.) LLC \\
\hline
\end{tabular}

Table 3. Five cases of proportional blending in the experiment.

\begin{tabular}{ccccc}
\hline Case & C1 & C2 & C3 & C4 \\
\hline Case1 & 1 & 1 & 1 & 1 \\
Case2 & 5 & 1 & 1 & 1 \\
Case3 & 1 & 3 & 1 & 3 \\
Case4 & 1 & 1 & 5 & 5 \\
Case5 & 5 & 3 & 2 & 1 \\
\hline
\end{tabular}

Four pure odorous chemical liquids were prepared and five liquid mixtures were carefully prepared using the four pure chemicals, in the proportions according to Table 3. All of these four pure liquids and five liquid mixtures were assembled in the odor dispenser. An odor can be reproduced in two different ways: (1) It can be reproduced by the proportional blending process using the four single pure liquids; or (2) it can be reproduced from a liquid mixture. After that, the electronic nose sampled odors reproduced either by the proportional blending of the four pure liquids or in one of the five mixed liquids. The monitoring procedure of the electronic nose was as follows:

1. The system atomizes an objective odor into an airtight chamber.

2. The chamber is kept static for $10 \mathrm{~min}$, so that objective odorants can be fulfilled in the chamber.

3. The electronic nose monitors the objective odor.

The monitoring procedure was executed 10 times, because each objective odor of the five cases was reproduced from either the proportional blending process or the odor liquid mixture. The electronic nose implemented 6 samples in each monitoring procedure. The working parameter settings of the electronic nose were as follows: Flush time was $120 \mathrm{~s}$; zero-point trim time was $10 \mathrm{~s}$; sampling interval was $5 \mathrm{~s}$; monitoring time was $120 \mathrm{~s}$; and dilution flow was $600 \mathrm{~mL} / \mathrm{min}$. As the electronic nose has 10 different types of gas sensors, as shown in Table 4, the data-set of each sample was a $120 \times 10$ matrix. It is noted that the fans were closed in the experiment and the chamber was sealed, with two tiny holes opened for the inlet of clean air and the outlet of the objective odor for the electronic nose. 
Table 4. The sensor array of the electronic nose.

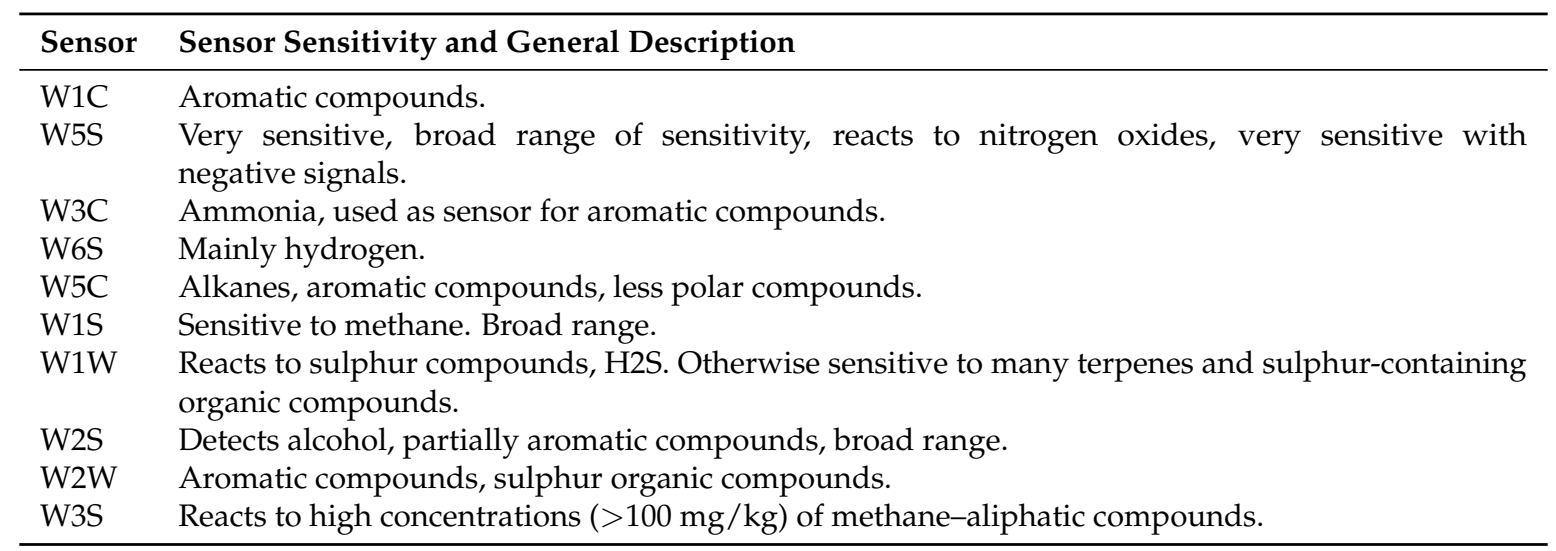

It was assumed that the response patterns sampled by the electronic nose were similar if odors generated in the two different ways were similar. Figure 7 shows the responses of the training dataset. It is clear that the response patterns of the five cases were relatively different. In feature extraction, we simply selected the 15th, 30th, 45th, and 60th response values from the samples, considering that little change occurred after $60 \mathrm{~s}$. The maximum response value and the differential values were extracted, as well. The dimensionality reduction was a combination of principal component analysis (PCA) and linear discriminant analysis (LDA). Pearson correlation was used for classification. Two principal components possessed a total of contribution of $99.48 \%$. Figure 8 shows that the classification was relatively good.
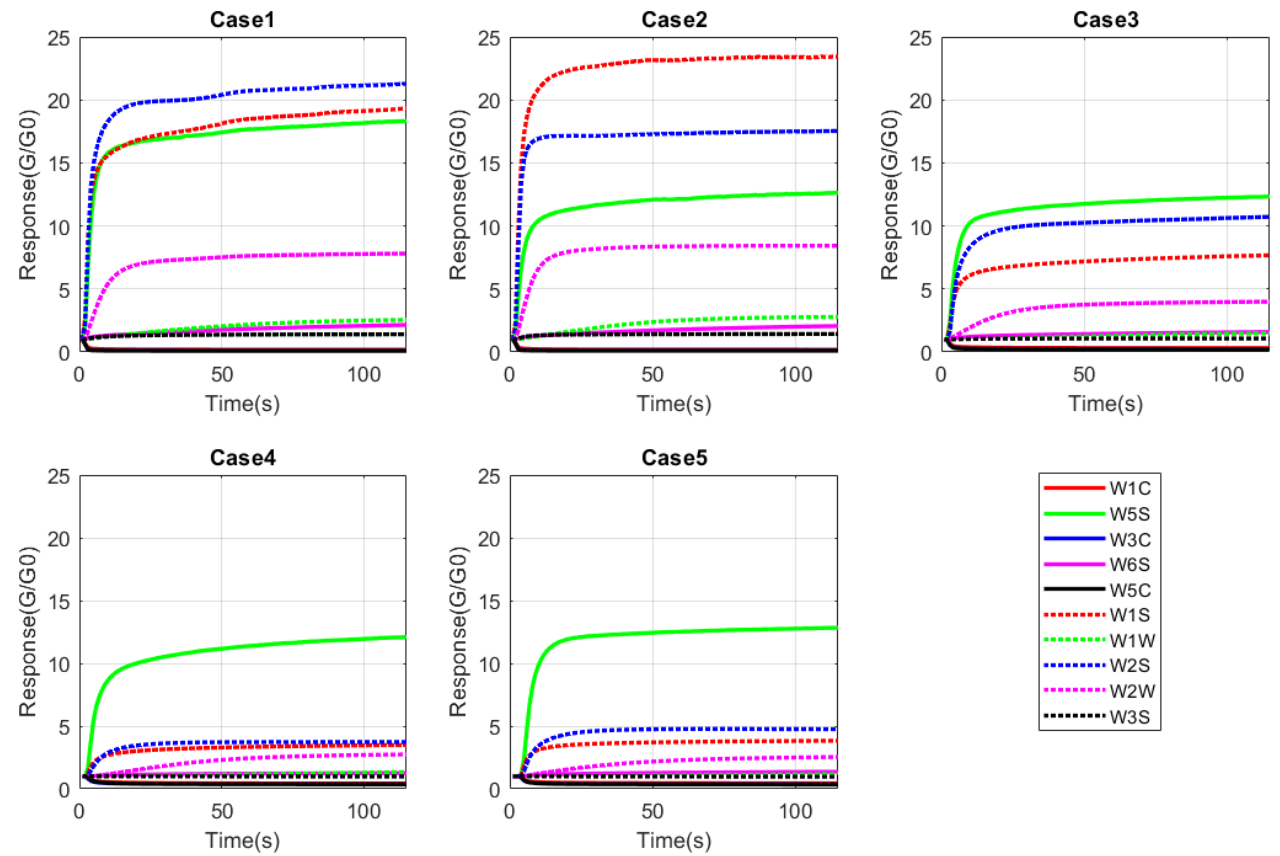

Figure 7. Sensory responses to the five cases. 


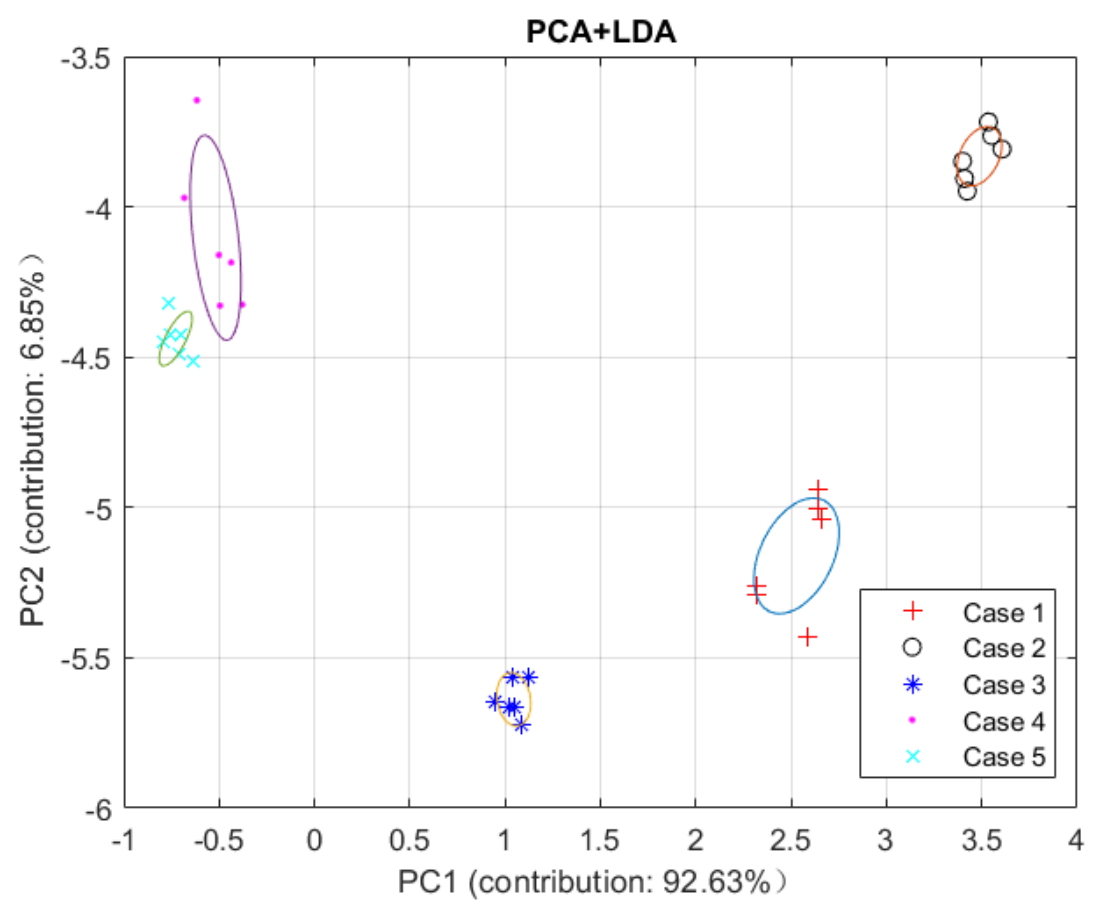

Figure 8. Classification of the five cases.

In the testing stage, responses sampled from the blending of four pure odorous chemicals were imported into the classification model. As shown in Table 5, the results of the first four cases were satisfactory, in that all identifications were correct. Only one sample was mismatched, in the fifth case. In summary, the total accuracy in all cases was $96.67 \%$, which was satisfactory to evaluate the effectiveness of reproducing odors.

Table 5. Five experiments of proportional blending in the experiment.

\begin{tabular}{cccc}
\hline Case & Correct Identification & Total Samples & Accuracy \\
\hline Case1 & 6 & 6 & $100 \%$ \\
Case2 & 6 & 6 & $100 \%$ \\
Case3 & 6 & 6 & $100 \%$ \\
Case4 & 6 & 6 & $100 \%$ \\
Case5 & 5 & 6 & $83.33 \%$ \\
Total & 29 & 30 & $96.67 \%$ \\
\hline
\end{tabular}

\section{Discussion}

Unlike an olfactory display, which represents an odor by releasing a prepared odor source, an odor reproduction system reproduces an odor by proportionally blending several odor sources. Accordingly, two components may significantly impact the performance of the system:

1. A careful selection of odor sources, and

2. Precise control of the generating odorants.

Odor sources (fragrance sources) are a fundamental element in an odor reproduction system, since the ultimate purpose of an ORS is to represent different odors. The mechanisms of olfactory perception are yet unclear in biology, neurology, and psychology [21]. A three-dimensional perceptual space can describe visible light, which has infinite frequencies in a narrow range of the spectrum, but the dimensionality of odor perceptual space is uncertain as of yet. J. B. Castro et al. [22] applied a non-negative matrix factorization (NMF) technique to derive a 10-dimensional olfactory space from 
the published data set of Dravnieks [23]. Meister suggested that the olfactory space may contain at most 20 dimensions [24]. Bushdid et al. estimated that human beings can discriminate over one trillion odors [25]. However, this was a controversial estimation [24,26]. Evidence has demonstrated that human beings may only discriminate at most 20 diverse odor percepts in an odor mixture [27]. This suggests that the number of odor sources used in an odor reproduction system may be limited. Moreover, the physical and chemical properties (oil solubility, water-solubility, volatility, viscosity, vapor pressure, or surface tension) of odor sources may lead to failure in blending odorants. Therefore, the odor sources used in an ORS must be carefully selected.

The proportion blending process is characteristic of an odor reproduction system. The quantities of various kinds of odorants should be precisely controlled, as this determines the odor intensity. Heating odor sources (liquid or solid) is one solution for generating odors [8]. Each odor source has a different evaporation rate, which is influenced by temperature, surface area, pressure, and airflow. The heating temperatures should be controlled below a certain threshold, to prevent scorching of the odor sources. The other way to generate odors is to atomize liquid sources by using piezoelectric transducers $[6,7]$. The atomizing quantity of odorants is determined by the size of transducers, the driving frequency, and the properties (surface tension, viscosity, and so on) of the odor sources. However, the problem that piezoelectric transducers fail to atomize liquids with higher viscosity arises. Moreover, the surface tension of a liquid impacts on the atomizing efficiency of a piezoelectric transducer [28].

Professional sniffers and odor identification instruments are two ways to evaluate the effectiveness of an odor reproduction system. Professional sniffers are panelists who can discriminate odors correctly. These panelists sniff an odor and give a mark. However, this requires professional training, which is a complicated and time-consuming task. Furthermore, human panelists are easily influenced by their bodily conditions and are sensitive to psychological fluctuations. The other way to evaluate an odor reproduction system is by using an odor identification instrument, such as Gas Chromatography-Mass Spectrometry (GC-MS), Gas Chromatography-Olfactometry (GC-O), or electronic nose instruments. Electronic noses have the advantage of short monitoring time and portability, which makes them suitable instruments for in-field monitoring [29]. An electronic nose with an array of gas sensors has the capacity for odor identification if the gas sensors are carefully selected and a suitable classification technique is used. In our study, a commercial electronic nose, PEN3, was applied to evaluate the performance of the proposed ORS. It revealed a satisfactory result, although a failure of identification happened. We suspected that the atomizing rates of each micro-porous piezoelectric transducer were slightly different, which influenced the accuracy of the proportion-blending process. Some sensors may have weak selectivity for odorous compounds. Moreover, a more suitable pattern recognition technique may increase the accuracy of identification, especially when an odor mixture contains more odorous chemicals.

The gas sensor component was embedded in the system for two reasons. First, the sensor can be used to monitor the cleanliness of the chamber. Second, it can also be used to measure each atomizing rate of the 16 micro-porous transducers, which is a problem requiring investigation. In the current design, a metal-oxide semiconductor (MOS) gas sensor TGS2600 is used. However, MOS-type sensors have a high selectivity for ethanol, which makes the identification of other chemicals in an odor mixture impossible [30]. In an odor reproduction system, the gas sensor should satisfy several requirements: Average sensitivity to various types of odorous compounds, corrosion resistance against some odorous compounds, and short response and recovery times.

\section{Conclusions}

Odor reproduction is a new area in machine olfaction with several challenges. Firstly, there is no appropriate model to build a practical odor reproduction system (ORS). Secondly, odor perception is a complicated subject, in which the relationship between odorous chemical compounds and odor perception needs to be investigated. Finally, precise control for the proportional blending is necessary in an ORS. In this paper, the authors proposed an ORS which has the capacity of reproducing odors by 
blending several odor sources in various proportions. The system contained a 16-channel odor dispenser using micro-porous piezoelectric transducers for atomizing odor liquids. A commercial electronic nose combined with a linear classifier was used to evaluate the effectiveness of the system. The training data were sampled from well-prepared odor mixtures and the testing data were sampled from odors which were reproduced by using several highly pure odor chemicals. The proposed ORS demonstrated a satisfactory result (96.67\% accuracy) in the identification. In the next step, some improvements to the system will be made and odor reproducing techniques will be further studied.

Author Contributions: Conceptualization, T.W. and D.L.; Formal analysis, T.W.; Methodology, T.W.; Project administration, D.L.; Software, T.W., Y.J., and P.Z.; Supervision, D.L.; Validation, T.W., Y.J., and P.Z.; Visualization, T.W., Y.J., and P.Z.; Writing—original draft, T.W.; Writing—review and editing, T.W.

Funding: The work was funded by National Natural Science Foundation of China grant number 61571140; Guangdong Science and Technology Department grant number 2017A010101032; and Guangdong Science and Technology Department grant number 2016A020226018

Conflicts of Interest: The authors declare no conflict of interest.

\section{Abbreviations}

The following abbreviations are used in this manuscript:

$\begin{array}{ll}\text { ORS } & \text { Odor reproduction system } \\ \text { PWM } & \text { Pulse width modulation } \\ \text { SAW } & \text { Surface acoustic wave } \\ \text { PS } & \text { Processing system } \\ \text { PL } & \text { Processing logic }\end{array}$

\section{References}

1. Gardner, J.W.; Bartlett, P.N. A brief history of electronic noses. Sens. Actuators B Chem. 1994, 18-19, $211-220$. [CrossRef]

2. Heilig, M.L.; Beach, L. Sensorama Simulator. U.S. Patent 3050870A, 28 August 1962.

3. Yanagida, Y.; Noma, H.; Tetsutani, N.; Tomono, A. An unencumbering, localized olfactory display. In CHI '03 Extended Abstracts on Human Factors in Computing Systems-CHI '03; Cockton, G., Korhonen, P., Eds.; ACM Press: New York, NY, USA, 2003; p. 988. [CrossRef]

4. Kim, D.W.; Cho, Y.H.; Nishimoto, K.; Kawakami, Y.; Kunifuji, S.; Ando, H. Development of aroma-Card based soundless Olfactory Display. In Proceedings of the 2009 16th IEEE International Conference on Electronics, Circuits and Systems-ICECS 2009, Yasmine Hammamet, Tunisia, 13-16 December 2009; IEEE: Piscataway, NJ, USA, 2009; pp. 703-706. [CrossRef]

5. Sugimoto, S.; Noguchi, D.; Bannnai, Y.; Okada, K. Ink jet olfactory display enabling instantaneous switches of scents. In ACM MM '10 \& Co-Located Workshops; del Bimbo, A., Chang, S.F., Smeulders, A., Eds.; Association for Computing Machinery: New York, NY, USA, 2010; p. 301. [CrossRef]

6. Abid, S.H.; Li, Z.; Li, R.; Waleed, J. Anaglyph video smell presentation using micro-porous piezoelectric film olfactory display. Displays 2015, 39, 55-67. [CrossRef]

7. Nakamoto, T.; Ito, S.; Kato, S.; Qi, G.P. Multicomponent Olfactory Display Using Solenoid Valves and SAW Atomizer and its Blending-Capability Evaluation. IEEE Sens. J. 2018, 18, 5213-5218. [CrossRef]

8. Covington, J.A.; Agbroko, S.O.; Tiele, A. Development of a Portable, Multichannel Olfactory Display Transducer. IEEE Sens. J. 2018, 18, 4969-4974. [CrossRef]

9. Wang, J.; Hu, H.; Ye, A.; Chen, J.; Zhang, P. Experimental investigation of surface acoustic wave atomization. Sens. Actuators A Phys. 2016, 238, 1-7. [CrossRef]

10. Kurosawa, M.; Watanabe, T.; Futami, K.; Higuchi, T. Surface acoustic wave atomizer. Sens. Actuators A Phys. 1995, 50, 69-74. [CrossRef]

11. Ariyakul, Y.; Nakamoto, T. Improvement of Odor Blender Using Electroosmotic Pumps and SAW Atomizer for Low-Volatile Scents. IEEE Sens. J. 2013, 13, 4918-4923. [CrossRef]

12. Howell, M.J.; Herrera, N.S.; Moore, A.G.; McMahan, R.P. A reproducible olfactory display for exploring olfaction in immersive media experiences. Multimed. Tools Appl. 2015, 75, 12311-12330. [CrossRef] 
13. Yamanaka, T.; Yoshikawa, K.; Nakamoto, T. Improvement of odor-recorder capability for recording dynamical change in odor. Sens. Actuators B Chem. 2004, 99, 367-372. [CrossRef]

14. Somboon, P.; Wyszynski, B.; Nakamoto, T. Study of odor recorder based on preconcentrator with variable temperature. Sens. Actuators B Chem. 2007, 127, 392-398. [CrossRef]

15. Somboon, P.; Kinoshita, M.; Wyszynski, B.; Nakamoto, T. Development of odor recorder with enhanced recording capabilities based on real-time mass spectrometry. Sens. Actuators B Chem. 2009, 141, 141-146. [CrossRef]

16. Harel, D.; Carmel, L.; Lancet, D. Towards an odor communication system. Comput. Biol. Chem. 2003, 27, 121-133. [CrossRef]

17. Rossiter, K.J. Structure-Odor Relationships. Chem. Rev. 1996, 96, 3201-3240. [CrossRef] [PubMed]

18. Poivet, E.; Peterlin, Z.; Tahirova, N.; Xu, L.; Altomare, C.; Paria, A.; Zou, D.; Firestein, S. Applying medicinal chemistry strategies to understand odorant discrimination. Nat. Commun. 2016, 7, 11157. [CrossRef] [PubMed]

19. Poivet, E.; Tahirova, N.; Peterlin, Z.; Xu, L.; Zou, D.; Acree, T.; Firestein, S. Functional odor classification through a medicinal chemistry approach. Sci. Adv. 2018, 4. [CrossRef] [PubMed]

20. Doty, R.L. Olfactory dysfunction in Parkinson disease. Nat. Rev. Neurol. 2012, 8, 329-339. [CrossRef] [PubMed]

21. Tromelin, A. Odour perception: A review of an intricate signalling pathway. Flavour Fragr. J. 2016, 31, 107-119. [CrossRef]

22. Castro, J.B.; Ramanathan, A.; Chennubhotla, C.S. Categorical dimensions of human odor descriptor space revealed by non-negative matrix factorization. PLoS ONE 2013, 8, e73289. [CrossRef]

23. Dravnieks, A. Atlas of Odor Character Profiles; ASTM Data Series, Vol. DS 61; ASTM: Philadelphia, PA, USA, 1985.

24. Meister, M. On the dimensionality of odor space. eLife 2015, 4, e07865. [CrossRef]

25. Bushdid, C.; Magnasco, M.O.; Vosshall, L.B.; Keller, A. Humans Can Discriminate More than 1 Trillion Olfactory Stimuli. Science 2014, 343, 1370. [CrossRef]

26. Gerkin, R.C.; Castro, J.B. The number of olfactory stimuli that humans can discriminate is still unknown. eLife 2015, 4, e08127. [CrossRef] [PubMed]

27. Weiss, T.; Snitz, K.; Yablonka, A.; Khan, R.M.; Gafsou, D.; Schneidman, E.; Sobel, N. Perceptual convergence of multi-component mixtures in olfaction implies an olfactory white. Proc. Natl. Acad. Sci. USA 2012, 109, 19959-19964. [CrossRef] [PubMed]

28. Ghazanfari, T.; Elhissi, A.M.A.; Ding, Z.; Taylor, K.M.G. The influence of fluid physicochemical properties on vibrating-mesh nebulization. Int. J. Pharm. 2007, 339, 103-111. [CrossRef] [PubMed]

29. Giungato, P.; Di Gilio, A.; Palmisani, J.; Marzocca, A.; Mazzone, A.; Brattoli, M.; Giua, R.; de Gennaro, G. Synergistic approaches for odor active compounds monitoring and identification: State of the art, integration, limits and potentialities of analytical and sensorial techniques. TrAC Trends Anal. Chem. 2018, 107, 116-129. [CrossRef]

30. Gebicki, J. Application of electrochemical sensors and sensor matrixes for measurement of odorous chemical compounds. TrAC Trends Anal. Chem. 2016, 77, 1-13. [CrossRef]

(c) 2019 by the authors. Licensee MDPI, Basel, Switzerland. This article is an open access article distributed under the terms and conditions of the Creative Commons Attribution (CC BY) license (http://creativecommons.org/licenses/by/4.0/). 